\title{
Activity-based differentiation of pathologists' workload in surgical pathology
}

\author{
G. A. Meijer • J. J. Oudejans • J. J. M. Koevoets • \\ C. J. L. M. Meijer
}

Received: 14 December 2007 /Revised: 17 March 2009/Accepted: 21 March 2009/Published online: 28 April 2009

(C) The Author(s) 2009. This article is published with open access at Springerlink.com

\begin{abstract}
Adequate budget control in pathology practice requires accurate allocation of resources. Any changes in types and numbers of specimens handled or protocols used will directly affect the pathologists' workload and consequently the allocation of resources. The aim of the present study was to develop a model for measuring the pathologists' workload that can take into account the changes mentioned above. The diagnostic process was analyzed and broken up into separate activities. The time needed to perform these activities was measured. Based on linear regression analysis, for each activity, the time needed was calculated as a function of the number of slides or blocks involved. The total pathologists' time required for a range of specimens was calculated based on standard protocols and validated by comparing to actually measured workload. Cutting up, microscopic procedures and dictating turned out to be highly correlated to number of blocks and/or slides per specimen. Calculated workload per type of specimen was significantly correlated to the actually measured workload. Modeling pathologists' workload based on formulas that calculate workload per type of specimen as a function of the number of blocks and slides provides a basis for a comprehensive, yet flexible, activity-based costing system for pathology.
\end{abstract}

G. A. Meijer $(\bowtie) \cdot J$. J. Oudejans $\cdot$ J. J. M. Koevoets $\cdot$

C. J. L. M. Meijer

Department of Pathology, VU University Medical Center,

Amsterdam, The Netherlands

e-mail: ga.meijer@vumc.nl
Keywords Pathology $\cdot$ Workload $\cdot$ Cost $\cdot$ Benchmarking

\section{Introduction}

To the outside world, including other hospital departments and hospital management, the pathology department often appears to function as a black box system with a specimen as input and a diagnosis as output. In reality, for each specimen, a complex number of activities are carried out at the macroscopic, microscopic, and molecular level. The kind and number of activities performed vary per type of specimen (e.g., liver biopsy, appendectomy, etc.), and are defined by protocols based on scientific evidence. Each of the individual activities (e.g., specimen reception, cutting up, microscopy, immunohistochemistry, electron microscopy, etc.) is to be carried out according to good laboratory practice (GLP) standards and requires the usage of resources like staff, consumables, and equipment. In turn, the type and amount of resources required varies per type of activity.

Obviously, the consumption of resources is not standard for each specimen, but depends on the type and number of activities required according to the protocol and carried out according to GLP guidelines.

Changes in types and numbers of specimens handled as well as changes of protocols (e.g., due to new clinical or scientific insights) will have direct consequences for workload. Successful management of a pathology laboratory requires a good insight in these processes and is essential for allocation of resources [1-5]. In the present study, we aimed to develop a model for measuring the pathologists' workload that can take into account such changes in types of specimens or protocols. 


\section{Materials and methods}

Study design

The hypothesis tested was that pathologists' workload (i.e., the work within the scope of the diagnostic process) can be measured and defined in an activity-based manner. This requires measuring workload (i.e., average time spent on a specimen) for an adequate series of cases of each diagnostic category occurring in a pathology practice. For common specimen types (e.g., gall bladder resection, breast lumpectomy, etc.), this is feasible; for many other less common types of specimens, this is a problem. However, when for different types of specimens the actual activities performed are listed (e.g., macroscopy: reading clinical data, describing macroscopic aspect of specimen, measuring size and weight, taking pictures, sampling frozen material, cutting blocks, etc.) appear to be common to many specimen types, albeit in different combinations and magnitude. Taking this approach, the diagnostic process for all specimen types occurring can be analyzed and broken down into separate activities (i.e., a product breakdown). So, times were scored not as a total time per type of specimen, but time per product breakdown activity; and in this way, adequate sample sizes can be obtained for each of these defined activities. Both macroscopic and microscopic procedures were broken down to separate activities. This also included communication by phone on individual cases. Clinicopathological conferences were not included. Examples of items scored for macroscopy and microscopy are given in Table 1.

Next, a list of specimen types occurring in our laboratory was constructed. This list consisted of 91 organ sites. Specimens were considered to be either biopsies or resections, thus yielding 182 specimen types. Importantly, all pathology diagnoses in the Netherlands are registered in the nationwide pathology registry PALGA, including SNOMED like codes for organ site and type of specimen [6]. Therefore, all individual laboratories in this way already document these relevant production variables.

\section{Measurements}

Second, the time needed to perform the product breakdown activities was measured. To this end, on consecutive days during a period of 12 weeks, a time-scoring form was added to each specimen received that day, and during the complete diagnostic process (from the inspection of the gross pathology to the final authorization of the report) the time required for each product breakdown activity was scored. During cutting up, times were scored by an independent observer; while during microscopy, time was scored by the pathologist for each product breakdown activity using a stopwatch. Data were provided by eight consultant pathologists and eight registrars. Times for consultants and registrars were scored and analyzed separately. In total, approximately 700 forms, covering a period of 12 weeks, were received and analyzed. In total, times for $11,200(700 \times 2 \times 8)$ product breakdown activities were scored.

\section{Data analysis}

Results of the measurements were entered in a specifically designed database. The data analysis aimed at finding a

Table 1 List of items in product breakdown of pathologists tasks that were scored individually for time needed during cutting up gross specimens and microscopy, respectively

\begin{tabular}{cl}
\hline Cutting up & \\
1 & Analyzing and dictating clinical information \\
2 & Consulting clinician \\
3 & Consulting supervisor \\
4 & Gross inspection and dictating \\
5 & Specimen photography \\
6 & Judging radicality of resection \\
7 & Painting specimens \\
8 & Dissecting resection margins \\
9 & Taking frozen sample/biobanking \\
10 & Weighing specimen \\
11 & Simple total embedding \\
12 & Split in half and embed \\
13 & Lamellate/multiple blocks \\
14 & Cut up and inspect \\
15 & Prepare for overnight fixation/pin down specimen \\
16 & Prepare for decalcification \\
17 & Exploring specimen (e.g., for harvesting lymph nodes) \\
Microscopy & \\
1 & Analyzing clinical information \\
2 & Retrieving archival slides from the patient \\
3 & Microscopy <5 slides H\&E \\
4 & Microscopy $5-10$ slides H\&E \\
5 & Microscopy $>10$ slides H\&E \\
6 & Requesting additional stains \\
7 & Microscopy of step sections \\
8 & Microscopy of special stains \\
9 & Microscopy of immunohistochemical stains \\
10 & Electron microscopy \\
11 & Quantitative pathology \\
12 & Deer consultation, literature searches \\
13 &
\end{tabular}

Time was only scored when items occurred, not all items occurred in all cases. In cases where consultant pathologists supervised registrars, relevant items (e.g. microscopy items) were scored twice, i.e., when registrars evaluated slides on their own as well as during supervision by the consultant pathologist 
relation between a specimen feature which is fixed (within certain limits) and the time cost per activity. The fixed variable used was the number of slides or tissue blocks. These data were based on the standard protocols used in our laboratory [7] and checked against the actual counting of the numbers of slides and blocks for the cases measured.

Using linear regression analysis, the relationship between the time needed for each activity, and the number of slides or blocks was analyzed. Based on this, for each activity, the time needed was calculated as a function of the number of slides or blocks involved, as prescribed by the protocol.

Given the calculated time per activity, the total pathologists' time required for a range of specimens was calculated based on the involved activities, again, as prescribed by the protocol.

To test the validity of this approach, for 22 specimen types that occurred with adequate frequency in the database, the correlation between these virtual specimen times and the actually measured times was computed. These 22 were biopsies of urinary bladder, bone marrow, uterine cervix, colon, rectum, endometrium, larynx, liver, stomach, duodenum, esophagus, breast, and prostate; and gross specimens of appendix, large intestine, gall bladder, lymph node, pancreas, ovary, placenta, prostate, and uterus. All statistical analyses were carried out using the SPSS 9.5 software.

\section{Results}

For all activities, significant correlations were found between the number of slides or the number of blocks and the time the pathologist needed for completing this activity. This was also the case in the situation where a pathologist was supervising a registrar. For instance, for the activities 'interpretation of H\&E slides', 'interpretation of extra stains' (i.e. PAS, deeper, etc.), and 'interpretation of immunohistochemical stains' the time required appeared to be significantly correlated to the number of slides involved in these activities (Fig. 1a-c). Interestingly, the graph for interpretation of H\&E sections slows a steeper
Fig. 1 Correlation between pathologist activities and number of slides involved. For the activities 'interpretation of H\&E slides', 'interpretation of extra stains' (i.e. PAS, step sections, etc.), and 'interpretation of immunohistochemical stains' the time required appeared to be significantly correlated to the number of slides involved in these activities (Fig. 1a-c). The time required for report dictating includes validation (Fig. 1day). Time is indicated as minutes and refers to times calculated for pathologists working on their own. The fact that the regression lines intercept the Y-axis at values $>0$ reflects the start-up time involved when beginning a new task. Similar analyses were performed for situations where pathologists supervised registrars (not shown)
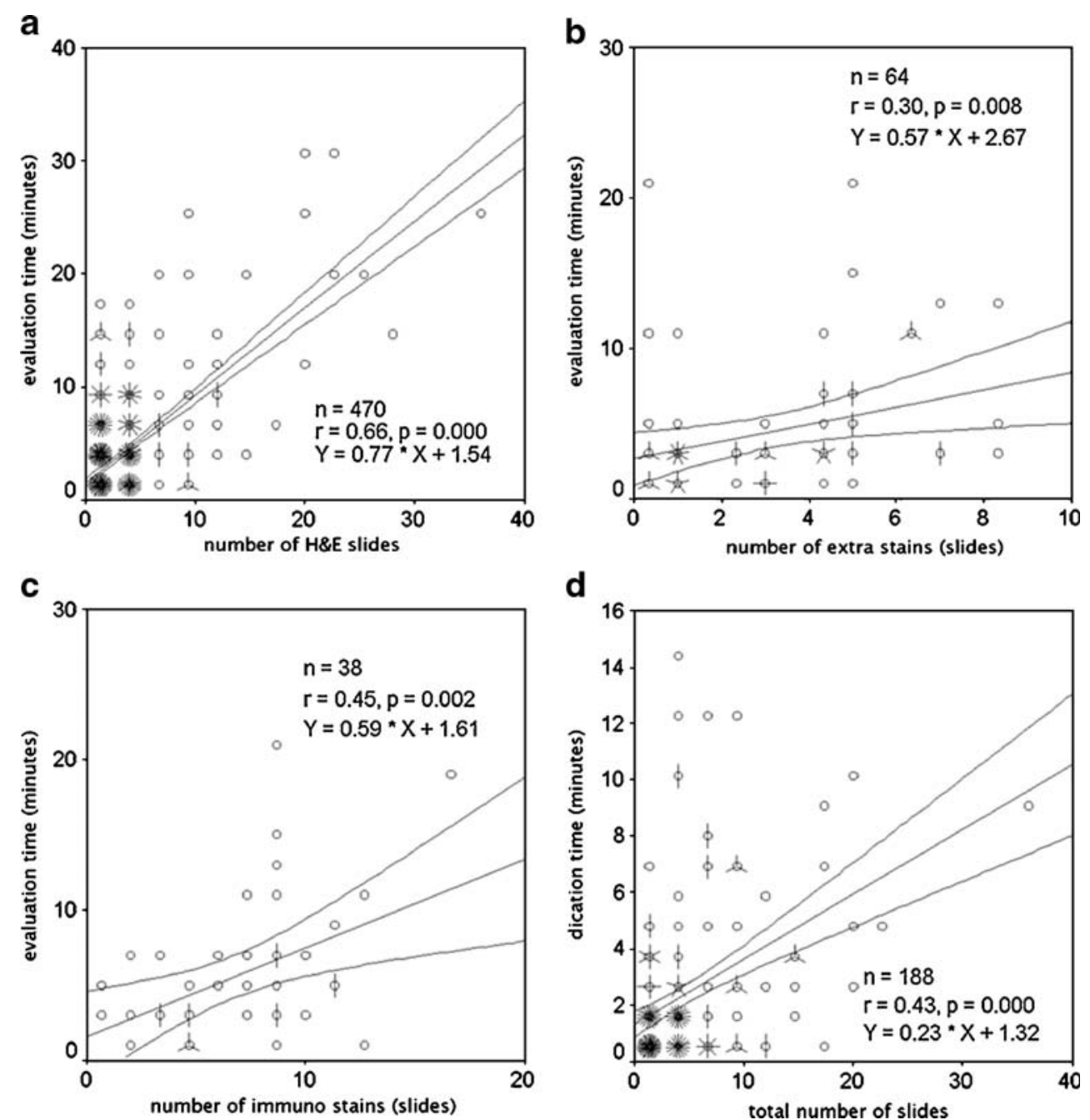

d

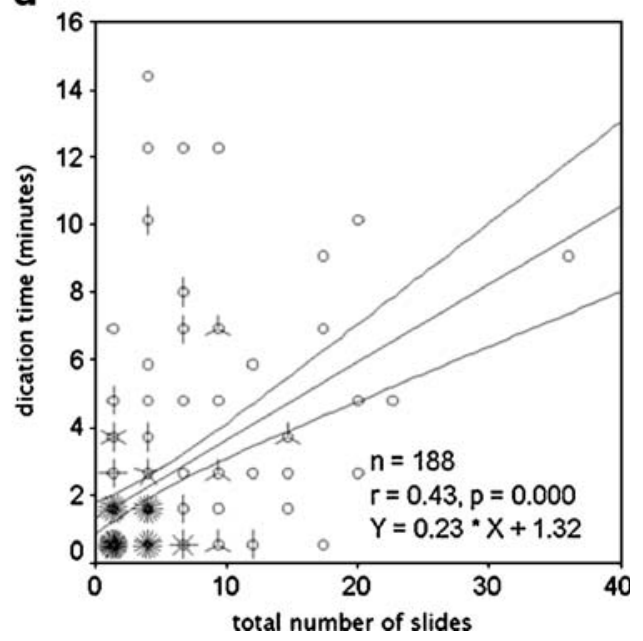


slope than the graphs for extra stains and immunohistochemical stains. This can be explained by the fact that when judging these latter stains, morphology of the lesions is already familiar to the pathologist from the H\&E sections and time is mainly spent on scoring histochemical or immunohistochemical features, which takes less time per slide. The time required for 'report dictating' was significantly correlated to the total number of slides per case (Fig. 1d).

Then, as described above, for all organs (as far as practically relevant), the virtual total pathologists' time involved was calculated from the regression function for each activity, and this result was multiplied with a factor that corrected for the frequency with which each activity occurred for every individual type of specimen. For example, in our laboratory, immunohistochemistry was applied in $10 \%$ of colorectal biopsies with an average of five slides. So, on average, for colorectal biopsies, the time involved for the activity 'evaluating immunohistochemical stains' is given by the equation $0.10 \times 5 \times A+B$, where $A=$ the factor of the regression function and $B$ the constant. Another example are bone marrow biopsies where immunohistochemistry is performed in $95 \%$ of cases with an average of eight markers and one negative control, which gives $0.95 \times 9 \times A+B$.

For 22 specimen types, enough actual measurements were present in the database to compare the calculated 'virtual pathologists' time' needed to evaluate and report a specimen with the times actually measured. Again, with linear regression analysis, the calculated times showed a high correlation with the actually measured total interpretation times. This was true for specimens that were handled by a consultant alone, and for specimens that were handled by a registrar who was supervised by a pathologist (Fig. 2a-b).

\section{Discussion}

Successful management of a pathology laboratory requires a good insight in these processes and is essential for allocation of resources. This insight is difficult mostly because pathologists find it difficult to obtain a realistic and quantitative economical perception of their everyday diagnostic practice. However, obtaining good insight is essential in particular in a period of increasing complexity of lab organization and technical processes with increasing costs. Here, we describe a comprehensive yet flexible approach for activity-based cost modeling that provides this insight in these processes and may be of help in mastering increasing budget constraints.

Knowledge of the workload that is involved with the products delivered is one of the basics of enterprise resource planning. Pathologists do not tend to regard their diagnostic reports as products, but from a management perspective, it is well defensible to compare the diagnostic process with a production process. From the specimen as raw material, using multiple resources, the pathologist makes a diagnosis, the end product. While nowadays, on one hand, the resources available are limited by budget constraints, the input (specimens) is not, neither in number nor in complexity. This may lead to serious budgetary problems. Budget control then is essential but this requires accurate information on the consumption of resources.

Medicine is a rapidly evolving profession, and this certainly goes for pathology. Several causes can be identified for the increase of pathologists' workload in the recent past. These include an increase in number of presented specimens as a result of an aging population with more possible cancer patients, and an ever increasing arsenal of diagnostic procedures available to the clinician continues to increase, and many of these, like new
Fig. 2 Validation of calculated time consumption versus actually measured time consumption. For 22 specimen types enough actual measurement were present in the database to compare the calculated 'virtual pathologist's time' with the actually measured times. The calculated times showed a high correlation with the actually measured times, both in the case the specimens were handled by a pathologist alone, as well as in those cases where the specimens were handled by a registrar who was supervised by a pathologist (Fig. 2a-b) a

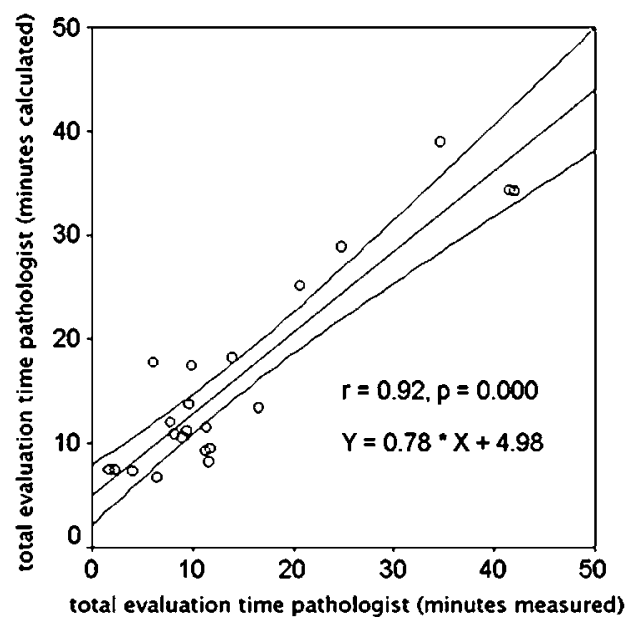

b

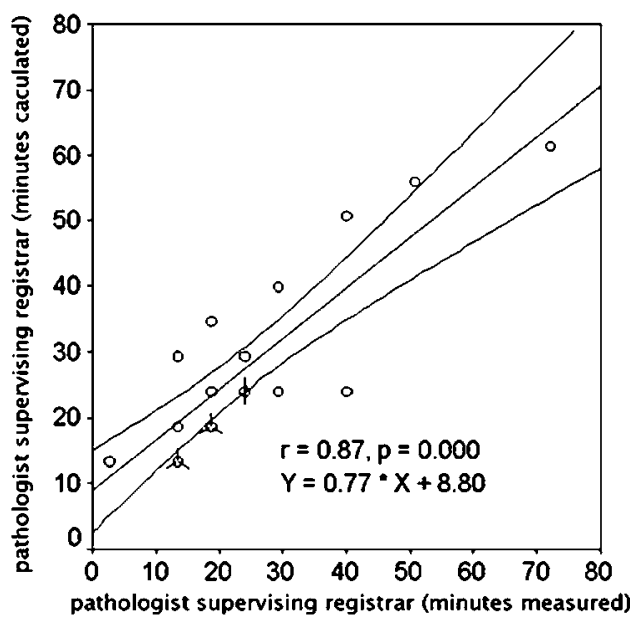


endoscopic techniques, allow for taking biopsies. In addition, rapid advances in biomedical science have tremendously increased our understanding of the biology of disease with considerably implications for clinical practice. A most prominent example is the development of targeted therapies in oncology (e.g., against her2neu, egfr, and c-kit), which require dedicated diagnostic procedures like immunohistochemistry, in situ hybridization, and mutation analysis. As a result, diagnostic reports become increasingly complex which leads to increasing demand for interdisciplinary meetings of clinicians and pathologists for discussing these cases.

Approaches to measuring pathologists' workload

The most simple approach is by just counting the number of specimens handled per period, but this has serious limitations. With an increase of laborious specimens, as discussed above, and a decrease of simple specimens, the total number of specimens may remain stable while the consumption of pathologists' resources has considerable increased. Also systems counting different diagnostic categories have their limitations $[2,4]$. Such trends can be detected when consumption of resources is measured in a differentiated way. Here, we present an activity-based, differentiated method to measure pathologists' labor, which is one of the major resources used in diagnostic pathology.

A protocol-based pathology practice, as is common in many institutions, enables to split the diagnostic process associated with different types of specimens in common activities, i.e. a product break down. However, in the same way as protocols should not be applied too rigidly, leaving room for a certain variation in the individual case and the individual pathologist, a workload measuring or costing system should be able to cope with variation. The system presented here was based on measuring a large number of cases handled by 16 different pathologists and registrars, thus covering a large variation in cases and pathologists. The results of the calculated workload correlated very well with the actual measurements, for individual activities as well as complete cases.

It should be noted, however, that the present study only focused on pathologists' workload, which in itself is largely insufficient to calculate a global budget of a lab. This requires an integrated approach that takes into account information on other resources (e.g., other staff like technicians, and secretaries, equipment, and consumables), to generate a model that, when fed with data of the actual numbers of the different types of specimens handled, can compute the theoretical budget in terms of staff, consumables, and equipment required to do the job. These data can be compared with the allowed budgets and the results could facilitate an optimized management of the pathology department.
Of course, real life is always more complicated than a simple computer model, but the aim of the current approach is not to produce virtual reality, but rather to have an adequate tool for workload and cost measurement in pathology. The method presented here is more flexible than the more classical approach of classifying cases in range groups of diagnoses, as is common in a number of countries. With the presented method, a change in the protocol is immediately reflected in the final calculations.

For implementing the presented approach in interested laboratories, several conditions should be met. The method used requires the coding of specimens. In our laboratory, histopathology specimens are coded according to site or organ (using a limited thesaurus) and type of procedure. As to the latter, we only use biopsy or resection. It turned out that in this way, the variation of workload within a certain type specimen was limited for almost all types of specimens. The major exception was skin pathology that showed a large variation in workload per specimen, and this variation did not segregate with biopsy or resection. To handle this point, skin specimens were divided in four groups (simple (naevi, basal cell carcinoma, etc.), inflammatory skin diseases, difficult differential diagnoses between benign and malignant, larger resection specimens (not difficult but many slides), and the mean workload for each group was calculated. Within these four groups, variation in workload per case was only limited. Moreover, groups 2 to 4 showed similar results so these could be taken together, leaving a group with "easy" skins and a group with "difficult" skins. For all four groups, again, a good correlation was found between the measured and calculated figures. To still allow the overall calculation of workload, an average time for skin specimens can be used applying a correction factor for the ratio of "easy" and "difficult" skins in a given department.

\section{Limitations of the present approach}

The present approach focused on measuring pathologists' workload, which is the production component that is most difficult to quantify. However, in itself, this is insufficient to calculate a global laboratory budget. For an adequate calculation of costs, it is also necessary to take into account the idle time lost during a working day. In an activity-based costing system, like the one presented here, this is not automatically included, in contrast to the "total absorption"based costing systems that primarily aim at recovering all costs made, but that are less suitable to detect changes in workload. So, in an activity-based costing system, a correction has to be included for idle time, e.g., by using a factor for the overall percentage of idle time. In addition, apart from surgical pathology, also other pathology tasks like cytology, autopsies, conferences, teaching, and re- 
search needs to be covered by the system. This can be done in way similar to that applied for surgical pathology. In principle, the methodology we used for calculating pathologists' workload can be implemented in every interested laboratory. The product breakdown items scored are listed in Table 1. The work reported here is limited to surgical pathology but we have applied similar strategies to cytology and post-mortem pathology (unpublished results).

In conclusion, we present an activity-based approach to measure pathologists' workload in surgical pathology that can be used as basis of a costing system. By applying formulas that calculate workload per type of specimen as a function of the number of blocks and slides, based on standard protocols, a reliable estimation of pathologists' workload per type of specimen can be made. With this approach, including also data on other resources like nonpathologist staff, consumables, and equipment, it is possible to have a comprehensive, yet flexible, activity-based costing system.

Acknowledgments The pathologists and registrars of the Department of Pathology, VU University Medical Center are acknowledged for their participation in performing the workload measurements.

Conflict of interest statement We declare that we have no conflict of interest.
Open Access This article is distributed under the terms of the Creative Commons Attribution Noncommercial License which permits any noncommercial use, distribution, and reproduction in any medium, provided the original author(s) and source are credited.

\section{References}

1. Freeman R (2002) Microbiology workload. J Clin Pathol 55:734

2. Parham DM (1996) The hidden increase in histopathologists' workload. J Clin Pathol 49:689-690

3. Riordan T, Cartwright K, Logan M et al (2002) How do microbiology consultants undertake their jobs? A survey of consultant time and tasks in South West England. J Clin Pathol $55: 735-740$

4. Tarbit IF (1990) Laboratory costing system based on number and type of test: its association with the Welcan workload measurement system. J Clin Pathol 43:92-97

5. Bellocq JP, Biron N, Kessler S et al (2001) Activity and cost analysis in surgical pathology. Experience of a French university laboratory using the activity-based costing method. Ann. Pathol. 21:215-232

6. Casparie M, Tiebosch AT, Burger G et al (2007) Pathology databanking and biobanking in The Netherlands, a central role for PALGA, the nationwide histopathology and cytopathology data network and archive. Cell Oncol 29:19-24

7. Anonymous (1995) Protocollenboek pathologie. Department of Pathology, Free University Hospital, Amsterdam, The Netherlands 